\title{
PERIODIC SOLUTIONS IN THE PROBLEM OF THREE BODIES *
}

BY F. H. MURRAY

1. Introduction. In Les Méthodes Nouvelles de la Mécanique Céleste, §48, Poincaré gave a discussion of the periodic solutions of the third kind in the problem of three bodies, which has formed the basis for later researches by Poincare and other writers. It is the purpose of this note to give an alternative demonstration of the existence of these solutions, using the methods of a preceding paragraph, and certain results of von Zeipel.

2. Statement of Problem. Suppose the number of degrees of freedom reduced to four by the methods of $\S 16$, $\dagger$ and the variables of $\S 18$ introduced. Then if

$$
\left\{\begin{array}{llll}
x_{1}=\Lambda, & x_{2}=\Lambda^{\prime}, & x_{3}=\xi, & x_{4}=\xi^{\prime}, \\
y_{1}=\lambda, & y_{2}=\lambda^{\prime}, & y_{3}=\eta, & y_{4}=\eta^{\prime},
\end{array}\right.
$$

the equations of motion become,

$$
\frac{d x_{i}}{d t}=\frac{\partial F}{\partial y_{i}}, \quad \frac{d y_{i}}{d t}=-\frac{\partial F}{\partial x_{i}}, \quad(i=1,2,3,4) .
$$

The function $F$ satisfies the relations

$$
\left\{\begin{array}{c}
F=F_{0}+\mu F_{1}+\cdots, \\
F_{0}=F_{0}\left(x_{1}, x_{2}\right), \quad \frac{\partial^{2} F_{0} \partial^{2} F_{0}}{\partial x_{1}{ }^{2}} \frac{\partial^{2} F_{0}}{\partial x_{2}}{ }^{2}-\left(\frac{x_{1} \partial x_{2}}{\partial x_{1}} \neq 0,\right.
\end{array}\right.
$$

in a domain $\left|x_{1}-x_{1}{ }^{0}\right|<b,\left|x_{2}-x_{2}{ }^{0}\right|<b$. Also,

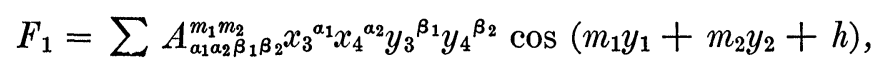

where $A_{a_{1} \alpha_{2} \beta_{1} \beta_{2}}^{m_{1} m_{2}}$ is analytic in $x_{1}, x_{2}$ in the domain considered.

We are now in a position to apply the method of $\S 46$. In the notation of that paragraph, the coordinates of a periodic

* Presented to the Society, September 8, 1922.

$\dagger$ Paragraph numbers refer to the paragraphs of Poincaré's Les Méthodes Nouvelles, vol. I. 
solution satisfy the conditions

$$
\begin{aligned}
\frac{\psi_{i}}{\mu}=\int_{0}^{T}\left(\frac{\partial F_{1}}{\partial y_{i}}+\mu \frac{\partial F_{2}}{\partial y_{i}}+\cdots\right) d t, \quad(i=1,2,3,4), \\
-\frac{\partial F_{0}}{\partial x_{i}{ }^{0}} T+\psi_{i}^{\prime}=-\int_{0}^{T} \frac{\partial F}{\partial x_{i}} d t, \quad(i=1,2), \\
\frac{\psi_{i}^{\prime}}{\mu}=-\int_{0}^{T}\left(\frac{\partial F_{1}}{\partial x_{i}}+\mu \frac{\partial F_{2}}{\partial x_{i}}+\cdots\right) d t, \quad(i=3,4), \\
\frac{\psi_{i}}{\mu}=\psi_{1}^{\prime}=\psi_{2}^{\prime}=\frac{\psi_{3}{ }^{\prime}}{\mu}=\frac{\psi_{4}^{\prime}}{\mu}=0, \quad(i=1,2,3,4) .
\end{aligned}
$$

These equations are to be solved for the values of the coordinates $\left(x_{i}, y_{i}\right)$, at the time $t=0$, and for $|\mu|<a$, where $a$ is arbitrarily small.

3. Solution of the Equations. On account of the integral $F=$ const., one of the equations, for instance $\psi_{2}=0$, may be omitted, and the constant $\bar{\omega}_{2}$ chosen identically zero. If $\mu=0$, equations (5) become

$$
\begin{gathered}
\frac{\partial R}{\partial \bar{\omega}_{1}}=\frac{\partial R}{\partial \bar{\omega}_{3}}=\frac{\partial R}{\partial \bar{\omega}_{4}}=\psi_{1}{ }^{\prime}=\psi_{2}{ }^{\prime}=\frac{\partial R}{\partial x_{3}{ }^{0}}=\frac{\partial R}{\partial x_{4}{ }^{0}}=0, \\
R=\frac{1}{T} \int_{0}^{T} F_{1} d t .
\end{gathered}
$$

The Jacobian of this system of functions is equal to the product of the Hessian of $F_{0}$ with respect to $x_{1}{ }^{0}, x_{2}{ }^{0}$ by the Hessian of $R$ with respect to $\bar{\omega}_{1}, \bar{\omega}_{3}, \bar{\omega}_{4}, x_{3}{ }^{0}, x_{4}{ }^{0}$, multiplied by a non-vanishing factor; from (3) it only remains to show that the latter, or the Hessian of $R$ with respect to $\lambda^{0}, \xi, \xi^{\prime}, \eta, \eta^{\prime}$, is different from zero if $x_{1}^{0}, x_{2}^{0}, \lambda^{0}$ are suitably chosen, and $\xi=\xi^{\prime}=\eta=\eta^{\prime}=0$.

This Hessian is equal, except for a non-vanishing factor, to the determinant $D_{2}$ of von Zeipel * (with $\theta=0$ ). It can easily be verified that for certain systems of values of $x_{1}{ }^{0}, x_{2}{ }^{0}$, $\lambda^{0}, D_{2} \neq 0$; the existence of periodic orbits of the corresponding types follows.

\section{The University of West Virginia}

* Recherches sur les solutions périodiques de la troisième sorte dans le problème des trois corps, Nova Acta R. Societ. Ups., (3), vol. 20, p. 51. 